\title{
Deutschlands Umweltindustrie fit machen für die Zukunft
}

Vor dem Hintergrund zahlreicher Umweltprobleme wird die Nachfrage nach Umwelttechnologien global stark ansteigen. Ein gezielter Ausbau der Technologieentwicklung in Deutschland verspricht neben den positiven Umweltfolgen, wirtschaftliche Gewinne und Entwicklungsperspektiven. Von Juliane Jörissen, Jens Schippl und Nora Weinberger

B evölkerungswachstum, Industrialisierung, Urbanisierung und steigender Wohlstand als gesellschaftliche Treiber des globalen Wandels haben zu globalen Umweltveränderungen wie Klimawandel, Bodendegradation, Abnahme der biologischen Vielfalt, Verknappung des Süßwasserangebots, Übernutzung und Verschmutzung der Weltmeere, Zunahme anthropogener Naturkatastrophen sowie einem steigenden Verbrauch natürlicher Rohstoffe geführt (WBGU 1999).

Um diesen Herausforderungen effektiv begegnen zu können, sind technische Entwicklungen und Durchbrüche unverzichtbar. Wie die Bundesregierung in ihrer Hightech-Strategie für Deutschland betont, lassen sich weder die Millenniumsziele der Vereinten Nationen noch die Ziele der deutschen Nachhaltigkeitsstrategie ohne den Einsatz effizienter, sogenannter sauberer Technik realisieren (BMBF 2006).

\section{Wachsende Märkte}

Der Markt für Umwelttechnologien ist gegenwärtig einer der global am stärksten wachsenden Märkte (BMU 2007; UBA/BMU 2007). Gerade für ein technologisch sehr fortschrittliches und zugleich exportorientiertes Industrieland wie Deutschland ergeben sich daraus große Chancen und Potenziale. Wie die Geschichte der europäischen Umweltschutzgesetzgebung zeigt, sind oftmals Regulierungen, die in einem Land zuerst angewandt wurden, später von anderen Ländern übernommen worden und haben dadurch den Unternehmen des Vorreiterlandes Exportvorteile verschafft. Auf diese Weise können Umweltregulierungen einen Leitmarkt für Umweltinnovation stimulieren (NIW/ZEW/ISI 2007).

Viele Hightech-Produkte, die für den europäischen Markt entwickelt wurden, sind für Entwicklungs- und Schwellenländer kaum bezahlbar und zudem unter den dort gegebenen institutionellen und organisatorischen Rahmenbedingungen häu- fig auch nicht sinnvoll einsetzbar. Es geht also auch darum, angepasste Technologien für den außereuropäischen Markt zu entwickeln. Der massive Energie- und Stoffdurchsatz der heutigen Industrieländer kann kein Leitbild für eine nachhaltige Weltwirtschaft sein. Besonderes Gewicht sollte deshalb auf die Möglichkeiten des „ökologischen Leapfrogging“ gelegt, also eine Entwicklung angeschoben werden, welche die nicht nachhaltigen Produktionstechnologien der Industrieländer überspringt (WBGU 2005).

Das komplexe Problem, Umwelttechnologien zur Bewältigung von Umweltproblemen zu entwickeln, sie an die Anforderungen von Schwellen- und Entwicklungsländern anzupassen, damit neue Märkte zu erschließen und gleichzeitig die Position Deutschlands im internationalen Wettbewerb zu verbessern, markiert die Herausforderung, vor der eine langfristig angelegte und strategisch orientierte Förderpolitik im Bereich der Umwelttechnologien steht. Die Unterstützung dieser Ausrichtung war zentrale Aufgabe des Projekts „Roadmap Umwelttechnologien 2020“.

\section{Langfristige Forschungsziele}

Vor dem skizzierten Hintergrund untersuchte das Projekt Roadmap Umwelttechnologien 2020 im Auftrag des Bundesministeriums für Bildung und Forschung, welche Beiträge Forschung und Technik für zukünftige Umweltinnovationen leisten können. Ziel des Projekts war es, strategische Optionen für die Forschungsförderung und die Unterstützung des Transfers in die Praxis aufzuzeigen. Es ging darum, zu analysieren, welche mittel- und langfristigen Entwicklungen in den Umwelttechnologien heute absehbar sind beziehungsweise welche hinsichtlich einer langfristigen Vorsorgeforschung aus heutiger Sicht als wünschenswert erscheinen. Dies erfordert die Kombination einer problemorientierten Vorgehensweise mit einer technikinduzierten Vorgehensweise. Der problemorientierte Ansatz fragt, wo vor dem Hintergrund der großen Umweltprobleme dringender Handlungsbedarf besteht und welche Technologien benötigt werden. Der technikinduzierte Ansatz fragt dagegen, welche Angebote und Möglichkeiten sich aus dem wissenschaftlich-technischen Fortschritt ergeben.

Das Projekt war in drei Phasen eingeteilt, die durch unterschiedliche methodische Ansätze charakterisiert sind. In Phase 1 wurde in einem Zwischenbericht der State-of-the-Art im Bereich Umwelttechnologien dargestellt. In Absprache mit dem Auftraggeber wurden sieben Handlungsfelder ausgewählt, die in Abbildung 1 dargestellt sind. Dazu wurden die drängend- $\rightarrow$ 


\section{„Der Einsatz von Umwelttechnologien stellt eine unverzichtbare Voraussetzung für eine verbesserte Lebenssituation und eine stabile wirtschaftliche Entwicklung dar."}

sten Umweltprobleme sowie entsprechende technische Lösungsoptionen aufgezeigt. Diese Arbeiten stützten sich auf eine umfangreiche Literaturrecherche, die durch Experteninterviews ergänzt und validiert wurde. An dieser Stelle sei erwähnt, dass Technologien zur Energiewandlung und -erzeugung nicht Gegenstand der Studie waren.

\section{Vier Themenkomplexe}

Auf der Basis des State-of-the-Art-Reports erfolgte in Phase 2 die Bildung von Hypothesen über wichtige Entwicklungen in der Umwelttechnik und, darauf aufbauend, die Auswahl von besonders relevant erscheinenden Technologiebereichen für eine breit angelegte schriftliche Expertenumfrage (Schippl et al. 2008; 2009). Für die Zwecke der Befragung wurden die im Projekt behandelten sieben Handlungsfelder $\mathrm{zu}$ vier Themenkomplexen zusammengefasst:

- Teil A: Wassermanagement

I Teil B: Klimaschutz und Luftreinhaltung

I Teil C: Bodenschutz, Erhalt der Biodiversität und Naturschutz

I Teil D: Erhöhung der Rohstoffproduktivität und Kreislaufwirtschaft

Jeder dieser Themenkomplexe enthielt etwa 20 funktionale Beschreibungen von Technologien, Verfahren oder Konzepten, denen aus Sicht des Projektteams zentrale Bedeutung zur Bewältigung der drängenden Umweltprobleme zukommt. Zu jedem Technologiebereich wurden sechs sich jeweils wiederholende Unterfragen gestellt, die sich auf die Bedeutung der betreffenden Technologie zur Lösung der Umweltprobleme, den Forschungs- und Förderbedarf, das Marktpotenzial, die Position Deutschlands im internationalen Vergleich sowie mögliche Hemmnisse für den erfolgreichen Einsatz der Technologie bezogen.

Neben den vier inhaltlichen Themenkomplexen gab es einen allgemeinen Teil, der drei übergreifende Fragen enthielt. Diese betrafen den Problemdruck in den sieben Umwelthandlungsfeldern global und national sowie den Bedarf an öffent- licher Förderung in Deutschland. Der Fragebogen wurde an circa 1.750 Experten aus Wissenschaft, Wirtschaft, Politik und Verwaltung verschickt, von denen etwa 440 geantwortet haben.

In Phase 3 wurden die vorläufigen Ergebnisse der Auswertung in vier themenspezifischen Workshops einem ausgewählten Kreis von Experten vorgestellt. Diese Workshops dienten sowohl der kritischen Auseinandersetzung mit den Umfrageergebnissen sowie als Forum für langfristig orientierte, eher visionäre Reflektionen über den Zeithorizont 2020 hinaus und bildeten damit den letzten Schritt der Datenerhebung. Die Ergebnisse des Projekts sind somit das Resultat eines ausgesprochen komplexen Prozesses der Erfassung, Aggregation und Auswertung von Expertenwissen unterschiedlicher Art, versehen mit mehreren Validierungsschleifen zur Sicherstellung von Objektivität und Transparenz.

\section{Problematik der Luftreinhaltung unterbewertet}

Die drei übergreifenden Fragen wurden von allen 440 Experten beantwortet. Die Ergebnisse der Antworten zur Frage nach dem Problemdruck sind in Abbildung 1 aufbreitet. In der gewählten Darstellungsform wurden die beiden höchsten Bewertungsoptionen aus einer fünfstufigen Skala von hoch bis gering aggregiert und in Prozent dargstellt.

Wie Abbildung 1 zeigt, sehen die Befragten den größten Problemdruck auf globaler Ebene in den Bereichen Wasser, Klima und Rohstoffe, was sich mit vielen anderen Studien sowie den Zielfeldern des Masterplans Umwelttechnologien der Bundesregierung deckt (BMU/BMBF, 2008). Aus nationaler Sicht steht der Klimaschutz an erster Stelle, gefolgt von dem Bereich Rohstoffe, während das Wassermanagement auf die unteren Ränge verwiesen wird. Der Problemdruck im Bereich Biodiversität und Naturschutz wird auf globaler Ebene höher eingeschätzt als auf nationaler Ebene, gleichwohl steht der Bereich im nationalen Ranking auf Platz drei, im globalen erst auf Platz fünf. Auch der globale Problemdruck im Bereich Luftreinhaltung wird sehr viel höher eingeschätzt als der nationale. Die Ergebnisse der schriftlichen Umfrage, sowohl zu den allgemeinen wie zu den technologiespezifischen Fragen, waren Gegenstand der Diskussion in den Experten-Workshops. Dabei wurde das Befragungsergebnis in vielerlei Hinsicht argumentativ gestützt, in einigen Bereichen aber auch hinterfragt. So deckten sich die Priorisierung der drei Top-Themen Klimawandel, Rohstoffe und Wassermanagement mit der Einschätzung der Workshop-Teilnehmer. Die relativ geringe Bedeutung, die dem Handlungsfeld Luftreinhaltung vor allem auf nationaler Ebene beigemessen wurde, konnten dagegen viele der Workshop-Teilnehmer nicht nachvollziehen. Ihrer Ansicht nach wird die Relevanz der Luftreinhaltung für den Gesundheits- und Umweltschutz im Vergleich zum heute allseits diskutierten Thema Klimawandel erheblich unterschätzt, auch mit Bezug auf Deutschland.

Verschiedene Teilnehmer äußerten zudem Verwunderung über die geringe Bedeutung, die dem Bereich der Abfallwirt- 
Abbildung 1: Wie schätzen Sie den Problemdruck global / in Deutschland in den nächsten zehn bis 20 Jahren in den folgenden Umwelthandlungsfeldern ein? (Die beiden höchsten Wertbereiche sind aggregiert und in Prozent dargestellt.)

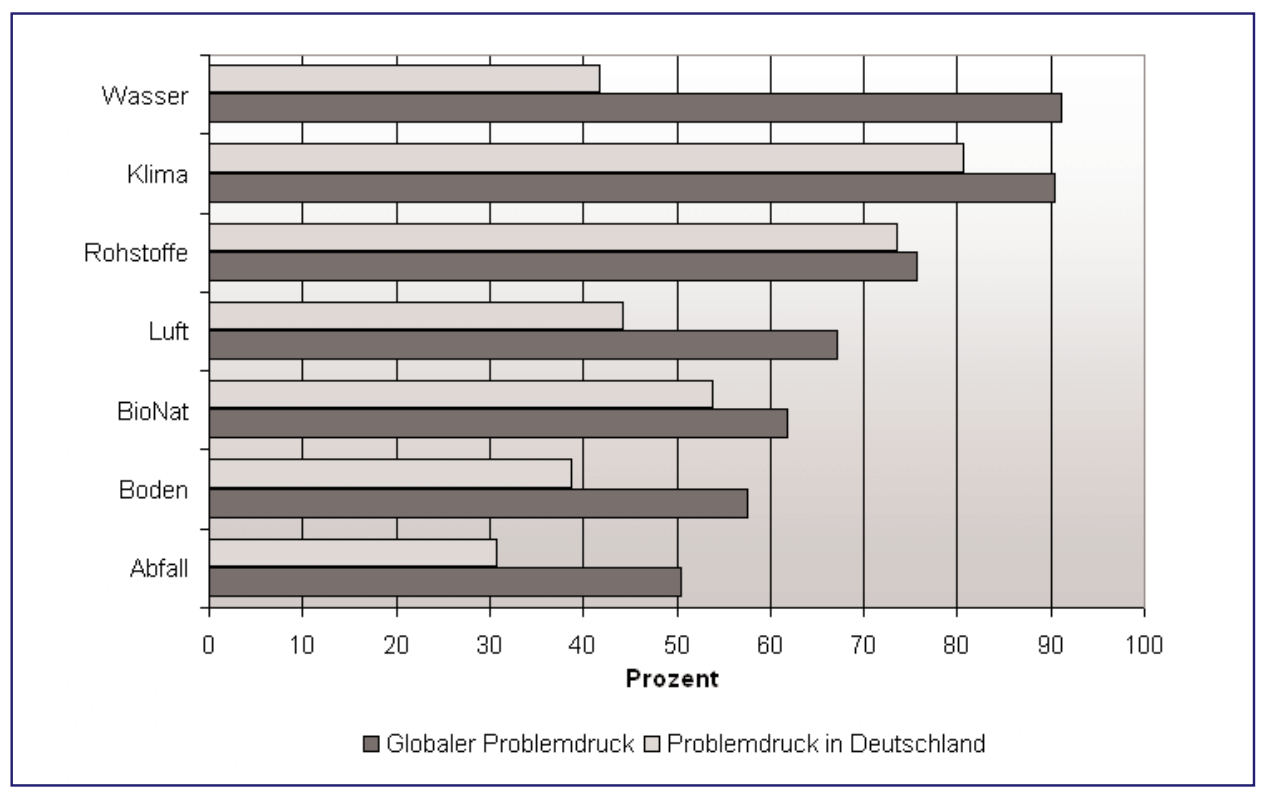

Quelle: Schippl et al. 2009

schaft in der Umfrage zugemessen wurde. Im Hinblick auf die Behandlung, Verbrennung und Verwertung von Abfällen, die unter dem Aspekt der Kohlenstoffdioxid- und Methan-Emissionen auch sehr klimarelevant sind, wurde auf globaler Ebene erheblicher Nachholbedarf gesehen. Für die weltweit anerkannte deutsche Abfalltechnik ergäben sich dadurch gute Vermarktungschancen. Der hohe globale Problemdruck im Bereich Wasser wurde im Workshop bestätigt. Allerdings wurde die relativ niedrige Gewichtung des Problemdrucks auf nationaler Ebene von den Teilnehmern nicht geteilt. Die Sicherstellung der Nahrungsmittelversorgung einer wachsenden Weltbevölkerung wurde gerade unter den Rahmenbedingungen des Klimawandels als ein zentrales Thema für die Zukunft betrachtet. Dazu gehören nach Ansicht der Workshopteilnehmer eine Intensivierung der landwirtschaftlichen Nutzung von Flächen, was einen erhöhten Wasserverbrauch nach sich zieht sowie die Anpassung an sich ändernde Niederschlagsverhältnisse. Erwartet wird daher eine starke Zunahme der Bewässerungslandwirtschaft in Europa und auch in Deutschland. In diesem Kontext wurden intelligente Bewässerungssysteme, Technologien zur Nährstoffrückgewinnung, Nutzungsoptimierungen und innovative Bewirtschaftungssysteme als interessante Themen für die Zukunft herausgestellt.

\section{Hohe Potenziale für Hightech-Lösungen}

Die allgemeinen Fragen dienten einer groben Einschätzung der Experten zu den Umwelthandlungsfeldern. Deutlich detailliertere Auskünfte zu den 77 ausgewählten Technologiebereichen gibt der umfangreiche, spezielle Teil der Befragung.
Diese Ergebnisse können hier nicht ausführlich dargestellt werden. Anknüpfend an die einleitend skizzierte aktuelle Diskussion zu den ökonomischen Perspektiven der Umwelttechnik sollen schlaglichtartig einige wichtige Resultate zu den geschätzten Marktpotenzialen präsentiert werden.

Abbildung 2 zeigt die Technologiebereiche, denen über alle Cluster hinweg die größten Marktchancen zugeschrieben werden. Dem Aufbau der Frage entsprechend wird dabei nach Industrieländern, Schwellenländern und Entwicklungsländern unterschieden. Zudem ist gekennzeichnet, welchem Cluster die Technologien zugehören. Dabei wird deutlich, dass die höchsten Marktpotenziale in den Industrieländern bei Technologien aus den Clustern Klimaschutz und Luftreinhaltung sowie Rohstoffproduktivität und Kreislaufwirtschaft gesehen werden. Dies ist insofern nachvollziehbar, als hier vornehmlich Hightech-Lösungen zur Geltung kommen, die auf die Märkte in hoch technisierten Gesellschaften zugeschnitten sind. Keine dieser Technologien findet sich bei den Schwellenländern oder bei den Entwicklungsländern unter den ersten acht.

\section{Technologieentwicklung in Entwicklungsländern}

Im Gegensatz dazu dominieren unter den ersten acht Technologiebereichen in den Entwicklungsländern die Technologien aus dem Bereich Wassermanagement. Erklären lässt sich dies durch die Trinkwasserknappheit und die agrarisch geprägten Wirtschaftssysteme in vielen Entwicklungsländern. Bei den Schwellenländern finden sich ebenfalls mehrere Technologien mit Bezug zur Landwirtschaft, so steht die bedarfsgerechte Bewässerung wie bei den Entwicklungsländern an oberster Stelle. In beiden Länderkategorien weist die standortangepasste Bodenbearbeitung auf die sehr hohe Relevanz des Problems Bodenerosion hin. Nur bei den Schwellenländern sind alle Cluster unter den ersten acht Technologien vertreten, allerdings mit unterschiedlichen Schwerpunktsetzungen. In den Industrieländern stehen im Cluster Klima und Luftreinhaltung Technologien im Vordergrund, bei denen es um den Aspekt der Energieeinsparung geht, beispielsweise mittels Leuchtmitteln, Wärmedämmung, Elektromotoren und Abwärmenutzung. In den Schwellenländern liegt der Fokus auf Technologien zu Adaption an den Klimawandel und zur solarthermischen Kühlung. 
Abbildung 2: Einschätzung des Marktpotenzials. Am höchsten bewertete Technologien (gewichtetes Mittel) für Industrieländer, Schwellenländer und Entwicklungsländer. A = Wassermanagement; $B=$ Klimaschutz/ Luftreinhaltung, $C=$ Bodenschutz/ Erhalt der Biodiversität/ Naturschutz, D = Erhöhung der Rohstoffproduktivität/ Kreislaufwirtschaft)

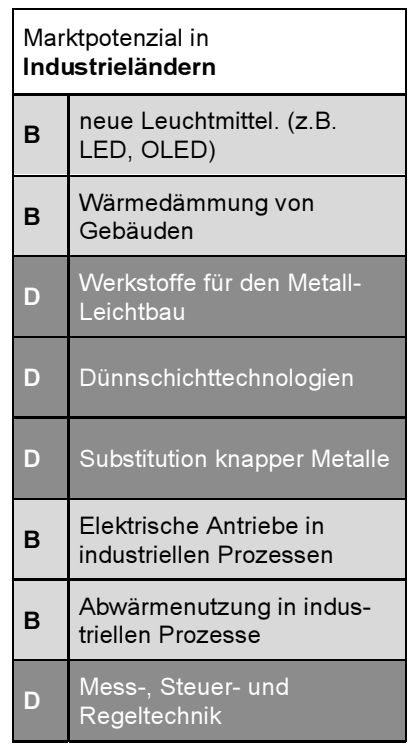

\begin{tabular}{|c|c|}
\hline \multicolumn{2}{|c|}{$\begin{array}{l}\text { Marktpotenzial in } \\
\text { Schwellenländern }\end{array}$} \\
\hline A & $\begin{array}{l}\text { Bedarfsgerechte } \\
\text { Bewässerung }\end{array}$ \\
\hline B & Adaptation an Klimawandel \\
\hline C & $\begin{array}{l}\text { Großflächiges Boden- } \\
\text { Monitoring }\end{array}$ \\
\hline B & Solarthermische Kühlung \\
\hline D & $\begin{array}{l}\text { Metallrückgewinnung aus } \\
\text { Abfällen }\end{array}$ \\
\hline A & $\begin{array}{l}\text { Meer- u. Brackwasser- } \\
\text { entsalzung mit reg. Energien }\end{array}$ \\
\hline D & $\begin{array}{l}\text { Sortierung und Aufbereitung } \\
\text { von Sekundärrohstoffen }\end{array}$ \\
\hline C & $\begin{array}{l}\text { Standortangepasste } \\
\text { Bodenbearbeitung }\end{array}$ \\
\hline
\end{tabular}

\begin{tabular}{|l|l|}
\hline \multicolumn{2}{|l|}{$\begin{array}{l}\text { Marktpotenzial in } \\
\text { Entwicklungsländern }\end{array}$} \\
\hline A & $\begin{array}{l}\text { Bedarfsgerechte } \\
\text { Bewässerung }\end{array}$ \\
\hline A & $\begin{array}{l}\text { Meer- u. Brackwasser- } \\
\text { entsalzung mit reg. Energien }\end{array}$ \\
\hline B & Adaptation an Klimawandel \\
\hline A & $\begin{array}{l}\text { Wasserspeicherkapazität } \\
\text { Böden (Bodenhilfsstoffe) }\end{array}$ \\
\hline A & $\begin{array}{l}\text { Chemikalienunabhängige } \\
\text { Wasseraufbereitung }\end{array}$ \\
\hline C & $\begin{array}{l}\text { Züchtung mehrjähriger Sorten } \\
\text { von Nutzpflanzen }\end{array}$ \\
\hline C & $\begin{array}{l}\text { Standortangepasste } \\
\text { Bodenbearbeitung }\end{array}$ \\
\hline A & \begin{tabular}{l} 
Aquakulturen \\
\hline
\end{tabular}
\end{tabular}

Quelle: Schippl et al. 2009

Die Ergebnisse zeigen, dass in der Regel den Technologien, bei denen hohe Marktpotenziale gesehen werden, auch ein hohes Problemlösungspotenzial zugeschrieben wird. Um weitergehende Aussagen zu ermöglichen, wurden die Marktpotenziale im Endbericht zu diesem Projekt im Zusammenhang mit den anderen in der Befragung abgefragten Parametern analysiert und interpretiert (Schippl et al. 2009). Die zentralen Ergebnisse sind in sogenannten Prioritätsfeldern zusammengefasst.

\section{Umwelttechnologien als Chance in der Krise}

Wie eingangs erwähnt, belegen verschiedene Studien die weltweit deutlich wachsende Nachfrage nach Umweltschutzgütern. Die Ergebnisse der vorliegenden Untersuchung demonstrieren, dass diese Aussage nicht nur durch eine ökonomische, innovationsorientierte Perspektive gestützt werden kann, sondern auch durch eine problemorientierte Betrachtung.

Der Blick auf zahlreiche ökologische Problemlagen mit ihren Einflussfaktoren und Implikationen zeigt, dass der Einsatz von Umwelttechnologien eine unverzichtbare Voraussetzung für eine Verbesserung der Lebenssituation und eine stabile wirtschaftliche Entwicklung darstellt. Das bedeutet, dass auch angesichts der aktuellen Wirtschaftkrise, die zunächst die Wirtschaftlichkeit mancher Umwelttechnologien in Frage zu stellen scheint, zumindest mittelfristig von einem steigenden Bedarf an Umweltschutzgütern ausgegangen werden kann.

\section{Literatur}

BMBF, Bundesministerium für Bildung und Forschung (Hrsg.): Die HightechStrategie für Deutschland. Berlin 2006
BMU, Bundesministerium für Umwelt, Naturschutz und Reaktorsicherheit / BMBF, Bundesministerium für Bildung und Forschung: Masterplan Umwelttechnologien. Berlin 2008.

BMU, Bundesministerium für Umwelt, Naturschutz und Reaktorsicherheit / UBA, Umweltbundesamt: Umweltpolitische Innovations- und Wachstumsmärkte aus Sicht der Unternehmen. Dessau 2007. Im Internet unter: http://www.umweltdaten.de/publikationen/fpdfl/3337.pdf

NIW, Niedersächsisches Institut für Wirtschaftsforschung / ZEW, Zentrum für Europäische Wirtschaftsforschung $\mathrm{GmbH}$ / ISI, FraunhoferInstitut für System- und Innovationsforschung: Zur technologischen Leistungsfähigkeit der deutschen Umweltschutzwirtschaft im internationalen Vergleich. Studien zum deutschen Innovationssystem Nr. 20-2007. Berlin 2006.

Schippl, J. / Grunwald, A. / Hartlieb, N. / Jörissen, J. / Mielicke, M. / Parodi, O. / Stelzer, V. / Weinberger, N. / Dieckhoff, C.: Roadmap Umwelttechnologien 2020. Zwischenbericht „State of the Art-Report“. Forschungszentrum Karlsruhe, Institut für Technikfolgenabschätzung und Systemanalyse. Karlsruhe 2008.

Schippl, J. / Grunwald, A. / Hartlieb, N. / Jörissen, J. / Mielicke, M. / Parodi, O. / Stelzer, V. / Weinberger, N. / Dieckhoff, C.: Roadmap Umwelttechnologien 2020. Endbericht. Forschungszentrum Karlsruhe, Institut für Technikfolgenabschätzung und Systemanalyse. Karlsruhe 2009.

UBA, Umweltbundesamt / BMU, Bundesministerium für Umwelt, Naturschutz und Reaktorsicherheit (Hrsg.): Wirtschaftsfaktor Umweltschutz Vertiefende Analyse zu Umweltschutz und Innovation. Dessau, München, Berlin 2007.

WBGU, Wissenschaftlicher Beirat der Bundesregierung Globale Umweltveränderungen: Welt im Wandel: Strategien zur Bekämpfung globaler Umweltrisiken. Jahresgutachten 1998. Berlin, Heidelberg, New York 1999.

WBGU, Wissenschaftlicher Beirat der Bundesregierung Globale Umweltveränderungen: Welt im Wandel. Armutsbekämpfung durch Umweltpolitik. Berlin, Heidelberg, New York 2005.

I AUTOREN + KONTAKT

Jens Schippl, Juliane Jörissen und Nora Weinberger sind wissenschaftliche Mitarbeiter(innen) am Institut für Technikfolgenabschätzung und Systemanalyse im Forschungszentrum Karlsruhe.

Forschungszentrum Karlsruhe, Institut für Technikfolgenabschätzung und Systemanalyse (ITAS), Postfach 3640, 76021 Karlsruhe. Tel.: +49 7247823994 , E-Mail: jens.schippl@itas.fzk.de, Internet: www.itas.fzk.de
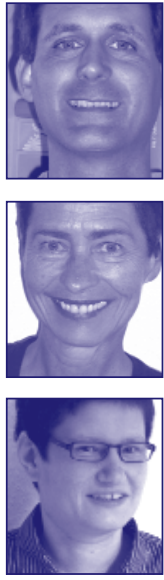
(c) 20I0 Authors; licensee IÖW and oekom verlag. This is an article distributed under the terms of the Creative Commons Attribution Non-Commercial No Derivates License (http://creativecommons.org/licenses/by-nc-nd/3.o/), which permits unrestricted use, distribution, and reproduction in any medium, provided the original work is properly cited. 